\title{
The Speed-Accuracy Tradeoff in the Elderly Brain: A Structural Model-Based Approach
}

\author{
Birte U. Forstmann, ${ }^{1}$ Marc Tittgemeyer, ${ }^{2}$ Eric-Jan Wagenmakers, ${ }^{1}$ Jan Derrfuss, ${ }^{2,3}$ Davide Imperati, ${ }^{2}$ and Scott Brown ${ }^{4}$ \\ ${ }^{1}$ Cognitive Science Center Amsterdam, University of Amsterdam, 1018 TV Amsterdam, Netherlands, ${ }^{2}$ Max Planck Institute for Neurological Research, \\ 50931Cologne, Germany, ${ }^{3}$ Radboud University Nijmegen, Donders Institute of Brain, Cognition and Behaviour, 6525 HR Nijmegen, Netherlands, and \\ ${ }^{4}$ Department of Psychology, University of Newcastle, Newcastle, New South Wales 2308, Australia
}

Even in the simplest laboratory tasks older adults generally take more time to respond than young adults. One of the reasons for this age-related slowing is that older adults are reluctant to commit errors, a cautious attitude that prompts them to accumulate more information before making a decision (Rabbitt, 1979). This suggests that age-related slowing may be partly due to unwillingness on behalf of elderly participants to adopt a fast-but-careless setting when asked. We investigate the neuroanatomical and neurocognitive basis of age-related slowing in a perceptual decision-making task where cues instructed young and old participants to respond either quickly or accurately. Mathematical modeling of the behavioral data confirmed that cueing for speed encouraged participants to set low response thresholds, but this was more evident in younger than older participants. Diffusion weighted structural images suggest that the more cautious threshold settings of older participants may be due to a reduction of white matter integrity in corticostriatal tracts that connect the pre-SMA to the striatum. These results are consistent with the striatal account of the speed-accuracy tradeoff according to which an increased emphasis on response speed increases the cortical input to the striatum, resulting in global disinhibition of the cortex. Our findings suggest that the unwillingness of older adults to adopt fast speed-accuracy tradeoff settings may not just reflect a strategic choice that is entirely under voluntary control, but that it may also reflect structural limitations: age-related decrements in brain connectivity.

\section{Introduction}

One of the most pervasive and ubiquitous effects of age is that it makes you slow. Even in the most elementary perceptual-cognitive tasks, older adults typically respond much more slowly than young adults. Why is this? One explanation is that age-related increases in neural noise slow down all processes related to evidence accumulation and response selection at the same rate (i.e., the global slowing hypothesis; e.g., Brinley, 1965; Cerella, 1985; Salthouse, 1996). From this perspective, age-related slowing stems from low-level neural deficiencies and is therefore both context-independent and unavoidable. A second explanation is that older adults are reluctant to make mistakes - for some reason, older adults attach more importance to responding accurately than to responding quickly (Rabbitt, 1979; Salthouse, 1979; Smith and Brewer, 1985, 1995; Starns and Ratcliff, 2010). Consequently, to avoid mistakes and reach near-perfect performance, older adults strike a balance between the opposing demands of accuracy and speed such that performance is relatively slow (Ratcliff et al., 2007, and references therein).

Received Jan. 18, 2011; revised 0ct. 8, 2011; accepted 0ct. 10, 2011.

Author contributions: B.U.F. designed research; B.U.F. performed research; B.U.F., M.T., E.-J.W., D.I., and S.B. analyzed data; B.U.F., M.T., E.-J.W., J.D., D.I., and S.B. wrote the paper.

This work was supported by VENI (B.U.F.) and VIDI (E.-J.W.) grants from the Netherlands Organization for Scientific Research (NWO). We thank Miriam Berger for the acquisition of the behavioral data, Raoul Grasman for helpful discussions, and one of the reviewers for suggesting pre-SMA exclusion mask procedure.

Correspondence should be addressed to Birte U. Forstmann Cognitive Science Center Amsterdam, University of Amsterdam, Plantage Muidergracht 24, 1018 TV Amsterdam, the Netherlands. E-mail: buforstmann@gmail.com. DOI:10.1523/JNEUROSCI.0309-11.2011

Copyright $\odot 2011$ the authors $\quad 0270-6474 / 11 / 3117242-08 \$ 15.00 / 0$
Because the speed-accuracy tradeoff (SAT) is highly nonlinear (Pew, 1969), a sizeable increase in response time can easily be accompanied by a modest increase in accuracy, such that an investment of 200 ms only yields a 3\% increase in percentage correct. This means that traditional methods of inference such as MANOVA may overlook the effects on accuracy and mistakenly conclude that SAT differences do not play a role. It is therefore essential to analyze the behavioral data with a mathematical model that explicitly accounts for the SAT. Whenever this has been done, results show that older adults are always more cautious than younger adults, and that it is only in a few specific tasks that older adults suffer a deficit in the quality of information processing (especially those tasks relying on perceptual discriminations; Ratcliff et al., 2007; Starns and Ratcliff, 2010). This line of work seems to support the notion that much of the slowdown with age is due to a strategic choice, a subjective and contextdependent preference to accumulate more evidence before committing to a decision.

The foregoing suggests that to better understand age-related slowing - and its neurological underpinnings - one needs to better understand the SAT and how it is implemented in the brain. Using fMRI, recent work has provided support for a striatal hypothesis of the SAT (Forstmann et al., 2008; Bogacz et al., 2010). This hypothesis states that speed stress increases the input from cortex to striatum; in turn, the striatum decreases the inhibitory control that is exerted by the output nuclei of the basal ganglia, disinhibiting the cortex and facilitating faster but possibly premature responses (Mink, 1996; Smith et al., 1998). The striatal hypothesis was supported by a recent study that used 


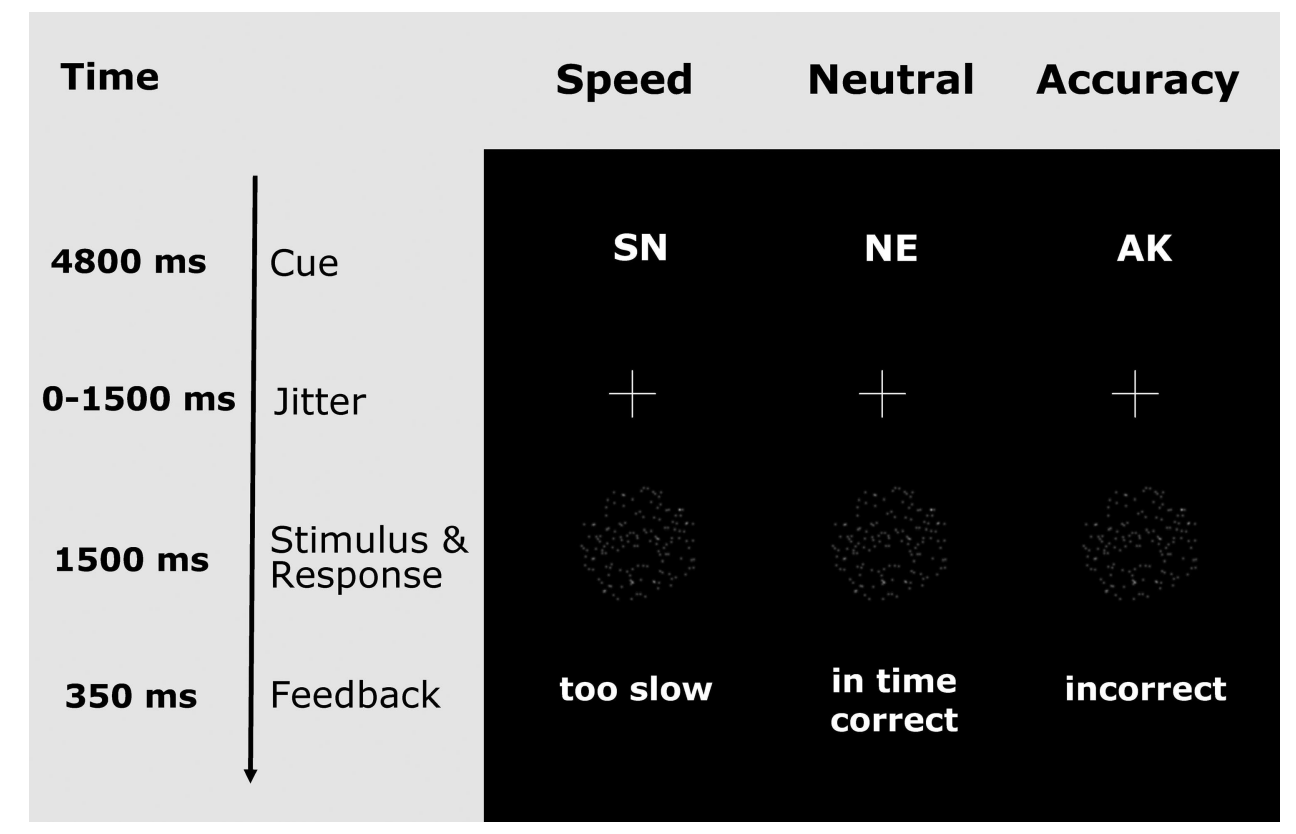

Figure 1. Paradigm. Moving dots paradigm with cues emphasizing Speed (SN for "schnell"), both speed and accuracy, that is, Neutral (NE), or Accuracy "AK" for "akkurat").

ultra-high resolution 7 Telsa (T) imaging in combination with structural diffusion-weighted imaging (DWI) to show that white matter tract strength between right pre-SMA and striatum is higher for participants who adjust their response thresholds more flexibly (Forstmann et al., 2010b).

Together, these theories suggest an interesting hypothesis: that age-related slowing might be caused at least partly by degeneration of corticostriatal connections. The goal of the current study was twofold; first, to use a mathematical process model of speeded decision making to confirm that age-related slowing can be partly attributed to more conservative threshold settings, and, second, to test the striatal hypothesis of the SAT, a hypothesis that predicts the strength of corticostriatal white matter connections to be compromised in the elderly. To pursue these goals we used a combination of techniques, most prominently mathematical modeling of behavioral data to quantify response caution (Brown and Heathcote, 2008) and DWI to quantify white matter connectivity between the brain regions of interest (Tuch et al., 2005; Cohen et al., 2009; Scholz et al., 2009; Forstmann et al., 2010b).

\section{Materials and Methods}

Participants. Twelve young subjects (eight women) and 12 elderly subjects (six women), all German native speakers, participated for a monetary reward of 8 euros. All participants signed a consent form before the scanning session. All participants had normal or corrected-to-normal vision, and none of them had a history of neurological, major medical, or psychiatric disorders. Young participants were on average 25.25 years old (range: 22-32; SD:3.47). Elderly participants were on average 64.50 years old (range: 61-79; SD: 5.05), were well educated and highly functioning, did not have a diagnosis of dementia or mild cognitive impairment, and did not have a history of hypertension, diabetes, or other conditions known to affect white matter integrity. All participants were righthanded, as confirmed by the Edinburgh Inventory (Oldfield, 1971). The study was approved by the local ethics committee and participants gave their written informed consent. Note that the behavioral and DWI data from the young subjects were reported by Forstmann et al. (2010b).

Behavioral task. In the present study we used the moving-dots task, popular in neuroscience and research with primates (Britten et al., 1992; for an overview see Gold and Shadlen, 2007). Participants were required to decide whether a cloud of dots appeared to move to the left or to the right (Fig. 1). Of 120 dots, 50\% moved coherently. Participants indicated their response by pressing 1 of 2 spatially compatible buttons with their left or right index finger. A cue (SN for "schnell," i.e., fast; NE for neutral; and AK for akkurat, i.e., accurate) instructed participants to adopt different levels of cautiousness on a trial-by-trial basis. The cues were pseudorandomly intermixed. At the end of each trial, participants received feedback that depended on the previously presented cue. In the speed and neutral condition, participants saw the message "zu langsam" (too slow) whenever they exceeded a response time criterion of 400 and $750 \mathrm{~ms}$ for the young participants, respectively, and 470 and $820 \mathrm{~ms}$ for the elderly participants, respectively. This choice was motivated by earlier work from Ratcliff and colleagues (Ratcliff et al., 2007), showing that elderly participants need more time for peripheral, non-decision processes than do younger participants (see also LBA model for response speed and accuracy). In the presence of such an age-related slowdown in peripheral processing, using the same response window for the old and the young participants produces more decision stress for the elderly participants. Thus, we aimed to equate the speed-stress for both age groups by explicitly taking into account the time needed for peripheral processing. Based on an earlier pilot study, we found that for the present task the age-related slowdown in peripheral processing is $\sim 70 \mathrm{~ms}$. We then added this amount to the response time window for the elderly participants.

In the accuracy condition, participants saw the message "falsch" (incorrect) whenever they made an incorrect response. This feedback procedure provided an additional incentive for participants to adopt different levels of response caution in response to the different cues. In total 840 trials, equally distributed over the 3 conditions and 4 blocks, were presented. The behavioral experiment lasted for $\sim 40 \mathrm{~min}$, yielding sufficient data for reliable estimation of the response boundary thresholds by using the LBA (Linear Ballistic Accumulator) model described below.

LBA model for response speed and accuracy. The reaction time data from correct and incorrect responses were modeled using the LBA model (Brown and Heathcote, 2008; see also Forstmann et al., 2008, 2010a,b). The LBA model represents a participant's choice task as a race between two independent accumulators corresponding to the two response options (illustrated below in Fig. 3A). On each trial, the two accumulators begin with random activation values drawn from independent uniform distributions on $[0, A]$. After the stimulus is presented, activation increases in each accumulator at a rate that depends on the stimulus. For 
example, activation will generally increase quickly in the accumulator whose response matches the stimulus, but slowly in the other accumulator. A response is triggered whenever the first accumulator reaches a fixed threshold $b$. The predicted response time is just the time taken for the first accumulator to reach the threshold, plus an offset parameter $\left(t_{0}\right)$.

Data acquisition and preprocessing of diffusion-weighted data. With an 8-channel array head coil and a maximum gradient strength of $40 \mathrm{mT} / \mathrm{m}$, diffusion-weighted data and T1-weighted images were acquired on a Siemens 3T Magnetom Trio scanner. The diffusion-weighted data were acquired using spin-echo echo planar imaging $(\mathrm{TR}=11 \mathrm{~s}$, $\mathrm{TE}=90 \mathrm{~ms}$, 85 axial slices, resolution $1.7 \times 1.7 \times 1.7 \mathrm{~mm})$. Diffusion weighting was isotropically distributed along 60 directions $(b$-value $=$ $1000 \mathrm{~s} / \mathrm{mm}^{2}$, number of excitations $=3$ ). Note that high angular resolution of the diffusion weighting directions yields robust probability density estimation by increasing the signal-to-noise ratio and reducing directional bias. Seven datasets with no diffusion weighting $(b 0)$ were acquired initially and after each block of 10 diffusion weighted images. These images served as an anatomical reference for offline motion correction. The acquisition of this protocol lasted $\sim 42 \mathrm{~min}$.

All baseline $b 0$ images were aligned to a reference $b 0$ image to estimate motion correction parameters using rigid-body transformations implemented in FLIRT (part of FSL software). The resulting linear transformation matrices were combined with a global registration to the T1 anatomy computed with the same method. The gradient direction for each volume was corrected using the rotation parameters. The transformation matrices were applied to the diffusion-weighted images, and the three corresponding acquisitions and gradient directions were averaged.

Tractography. In accordance with Behrens et al. (2003), estimation of white matter tracts was conducted using probabilistic tractography. This technique allows one to estimate white matter connections between brain regions of interest. Differences in white matter connectivity may originate from differences in axon caliber or neural myelination (Fields, 2008; Beaulieu, 2009), suggesting that the structural connectivity measures may provide partial information about the functional effectiveness of fiber bundles (because bundles with a higher degree of myelination are able to process information more rapidly).

Diffusion image preprocessing and analyses was done using FSL 4.1.4 (www.fmrib.ox.ac.uk/fsl). A probabilistic fiber tracking approach was chosen, using 5000 tract-following samples at each voxel with a curvature threshold of 0.2. A dual-fiber model as implemented in the latest version of bedpostX (FSL 4.1.4) was used. Dual-fiber models account for crossing fibers (Behrens et al., 2007), therefore yielding more reliable results compared with single-fiber models. All tractography was done in each participant's native space (un-normalized) data, and resulting maps were warped into standard space (using the MNI $1 \mathrm{~mm}$ isotropic brain as reference) for cross-participant averaging and comparison. For the estimation of tract strength between the left and right striatum as well as left and right pre-SMA, respectively, MNI-space masks were normalized to each participant's native space, using the inverse of the normalization parameters. Visual inspection ensured that tractography maps were acceptable for further analysis.

Seed-based classification was done by first thresholding the images such that only voxels with at least 10 samples were kept (Aron et al., 2007). Next, voxel values were converted into proportions, such that the value at each voxel became the number of samples reaching the target mask for that image, divided by the number of samples from the seed mask. Seed-based classification was done from the left and right pre-SMA into the left and right striatum, respectively (see Fig. 4). The values were later used for correlations with the mathematical model parameters reflecting response caution. All analyses were done separately for each hemisphere.

Cortical and subcortical target regions. A priori masks were chosen to compute the connectivity of areas within the cortiocstriatal network (see Fig. 4). The pre-SMA mask was defined with rostrocaudal boundaries ranging from $y=0$ to $y=30$, based on the mask used by Johansen-Berg et al. (2004). The striatal mask was taken from the Harvard-Oxford structural atlas implemented in FSL (www.fmrib.ox.ac.uk/fsl/data/atlasdescriptions.html\#ho). We estimated the strengths of connections between several regions chosen on the basis of the anatomical and neurocomputational hypotheses. In particular, we estimated the strength of connection between the left and right pre-SMA and left and right striatum, as these connections were proposed to mediate control over SAT by the striatal hypothesis (Bogacz et al., 2010; Forstmann et al., 2010b).

In the connectivity analysis of the above four brain regions it is possible that a tract starting in, say, the left pre-SMA would first connect to the right pre-SMA and then to the right striatum; this could falsely suggest a contralateral direct connection from left pre-SMA to right striatum. To prevent such spurious contralateral connections, we used a pre-SMA exclusion mask; that is, tracts starting in the left pre-SMA were not allowed to go first to the right pre-SMA and tracts starting in the right pre-SMA were not allowed to go first to the left pre-SMA.

\section{Results}

\section{Behavioral data}

We obtained the typical pattern of speed-accuracy results for both young and elderly participants, summarized in Figure 2. Under instructions to respond more quickly, mean response time (RT) was significantly shorter at the cost of more errors for both younger and older participants. This effect is most pronounced for the transition to the speed condition, that is, behavioral data from the neutral condition were quite similar to those from the accurate condition, but neither of these conditions was behaviorally similar to the speed condition. The older participants responded more slowly than younger participants in all conditions (main effect of group: $F_{(1,23)}=15.3 ; p=0.001$ ). The corresponding pattern was observed in error rates: across the three conditions, older participants committed more errors than younger participants $F_{(1,23)}=4.23, p=0.05$. No reliable interaction was found for either mean RT $(F<1.24, p=0.30)$ or error rates $(F<$ $0.23, p=0.64)$. Note that accuracy is well above chance levels in all conditions and for both groups, showing that participants did not resort to guessing.

\section{LBA model for the behavioral data}

The LBA model has five parameters that determine its predictions for a pair of correct and incorrect response time distributions. However, constraint is gained by fixing many of these parameters across experimental conditions (see Donkin et al., 2011, for extensive discussion of these methods). For example, in the absence of prior response biases, the response threshold parameter $b$ should be equal for left and right moving stimuli. Equally, one might expect the response threshold parameter to be different across the types of cue (speed, neutral and accuracy) as these were intended precisely to manipulate response caution.

\section{LBA effect of cue type (i.e., SAT instruction)}

We investigated eight different designs for constraining the parameters of the LBA model across cue type. The eight different designs consisted of all combinations allowing three model parameters to either vary with cue type, or be fixed across cue type. 
Table 1. Average LBA model parameter estimates for the older and younger participants

\begin{tabular}{lcr}
\hline & Elderly & \multicolumn{1}{l}{ Young } \\
\hline $\begin{array}{l}\text { Mean drift rate: accumulator corresponding to the } \\
\quad \text { correct response }\end{array}$ & $3.05(0.18)$ & $2.53(0.13)$ \\
$\begin{array}{l}\text { Mean drift rate: accumulator corresponding to the } \\
\quad \text { incorrect response }\end{array}$ & $1.57(0.23)$ & $0.69(0.19)$ \\
Size of start point distribution $(A)$ & $1.33(0.13)$ & $0.73(0.079)$ \\
Non-decision time $\left(t_{0}\right)$ & $0.292(0.028)$ & $0.286(0.011)$ \\
Response threshold $(b$, accuracy condition) & $1.86(0.24)$ & $0.976(0.094)$ \\
Response threshold $(b$, neutral condition) & $1.71(0.21)$ & $0.914(0.091)$ \\
Response threshold $(b$, speed condition) & $1.36(0.15)$ & $0.76(0.792)$ \\
\hline Numbers in parentheses show SEs under the assumption of normal & &
\end{tabular}

Numbers in parentheses show SEs under the assumption of normal sampling distributions.

The three parameters tested in this way were the response threshold parameter $(b)$, the drift rate parameter $(v)$ and the time taken for non-decision processes $\left(t_{0}\right)$. We made the simplifying assumption that the variability of the start point distribution should be fixed between speed and accuracy conditions (i.e., parameter $A$ ). This assumption represents standard practice when modeling speed-accuracy tradeoffs (Ratcliff and Rouder, 1998). The variability of the drift rate distribution was arbitrarily fixed at $s=1$, to satisfy a mathematical scaling property of the model, and also held constant across conditions.

For each of the eight model constraint designs, we estimated parameters using the method of maximum likelihood. Start points for SIMPLEX searches (Nelder and Mead, 1965) were generated using automatic heuristics. For all models except the simplest, extra parameter searches were run using start points generated from the best-fitting parameters for simpler, nested models. The best-fitting parameters were used to calculate BIC measures of model adequacy (Schwarz, 1978) for each of the eight designs, separately for each participant.

The design with the best BIC grouped across participants (i.e., BIC calculated with log-likelihood, number of parameters, and sample size all summed across participants) allowed only the response threshold parameter $(b)$ to vary with cue type, keeping all other parameters fixed. This confirms that the experimental manipulation of urgency cues successfully influenced participants to change the amount of evidence they required to make a decision, without influencing other aspects of the decision process. To quantify confidence in this result, we used the BIC measures to approximate posterior model probabilities for the different parameter constraint designs. This approximation is conditional on the true model being one of the comparison set (Burnham and Anderson, 2002), and also assumes a fixed-effects model for participants. Under this analysis, the evidence in favor of the design above (allowing only parameter $b$ to vary with cue type) was overwhelming: the posterior model probability for this model was $10^{19}$ times greater than for its nearest competitor. Such extreme results are typical when fixed-effect analyses are used for data that actually include random variability in effect size, and so-as an overly conservative precaution-we also applied the same analysis separately to each individual subject. This assumption is overly conservative because, for each individual subject, the assumption of a fixed effect is trivially true. We found that, averaged over subjects, the posterior model probability for the above design was $44 \%$, which was more than three times more probable than the next-best model.

Table 1 shows the average parameter estimates, across participants, separately for the elderly and younger groups. These values showed that the effect of cue type was quite strongaverage response thresholds were set at $37 \%$ or $27 \%$ more than the minimum possible value in response to an "accurate" or "neutral" cue, but only at $0.9 \%$ more than the minimum value in response to a "speed" cue (the minimum possible value for the response threshold is the upper limit of the start point distribution, parameter $A$ ).

\section{Model fit}

With the severe parameter constraints (only threshold varying across conditions), Figure $3 \mathrm{~B}$ shows that the model fits the data well. Instead of mean response times and error rates, this time we summarize both response accuracy and the full response time distributions for correct and incorrect responses in each condition. Each distribution is summarized by five quantiles that estimate the associated cumulative distribution functions (for a detailed explanation, see Donkin et al., 2011).

The quantile estimates support a detailed inspection of the data. For example, in the speed emphasis condition, the quantile estimates for the incorrect response times were all faster than the corresponding quantile estimates for correct response times, which replicates the usual finding that errors are fast, when speed is stressed (Ratcliff and Rouder, 1998). These results apply to both young and elderly participants. When accuracy was stressed, error response times tended to be approximately equal to correct response times, but also a little more variable (there was a greater separation between the quantile estimates). Comparisons of the data (circles in Fig. 3B) with model predictions (Fig. 3B, lines and crosses) show that the model fits the data very well. The predicted response probabilities are within $2.8 \%$ of the observed values for all conditions, and the predicted response quantiles for correct responses are always within $28 \mathrm{~ms}$ of the observed quantiles for correct responses and $88 \mathrm{~ms}$ for the incorrect responses.

In sum, application of the LBA model confirms that the behavioral effects of the experimental SAT manipulation can be well accounted for by a nothing more than a change in the response threshold. Importantly, this is true for both young and elderly participants. We now use the LBA to identify the locus of performance differences between young and elderly participants.

\section{LBA effects of aging}

Two important LBA parameters that are outside the focus of this work are those two that were not found to change with speed- vs accuracy-emphasis: non-decision time and drift rate. The average non-decision time was only $6 \mathrm{~ms}$ faster for younger than older participants, although this was influenced by one improbably fast estimate for an older participant (e.g., the difference in median non-decision time between the groups was larger, at $24 \mathrm{~ms}$ ). Nevertheless, this difference is smaller than the difference expected from prior research $(70 \mathrm{~ms})$; however, discrepancies are to be expected in small samples such as ours.

The younger and older groups also differed in their mean drift rate estimates. The mean drift rates for both the accumulator corresponding to the correct response and the accumulator corresponding to the incorrect response were significantly larger for the older than the younger participants $(p=0.007$ and $p=0.03$, respectively). In contrast, the best measure of information quality, the difference in drift rate between the two accumulators, was larger for the younger than the older participants, but this difference was not statistically significant $(p>0.1)$. These parameters fit with the idea that older people sometimes engage more carefully with the task and pay greater attention (higher drift rates), do not suffer any great decrement in the quality of decision information (nonsignificant discrepancy in the difference in drift 
A

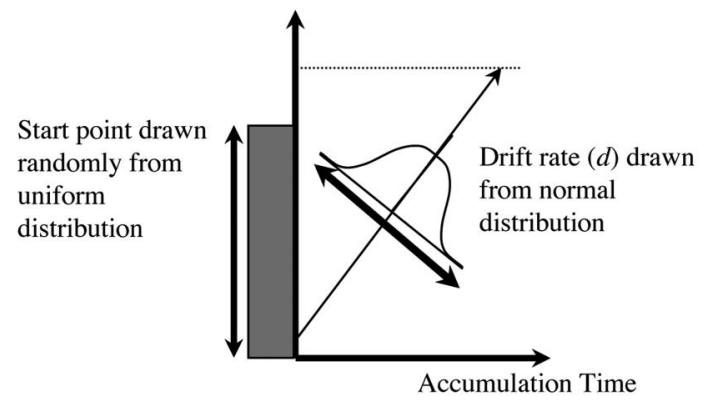

Evidence for

Right Response

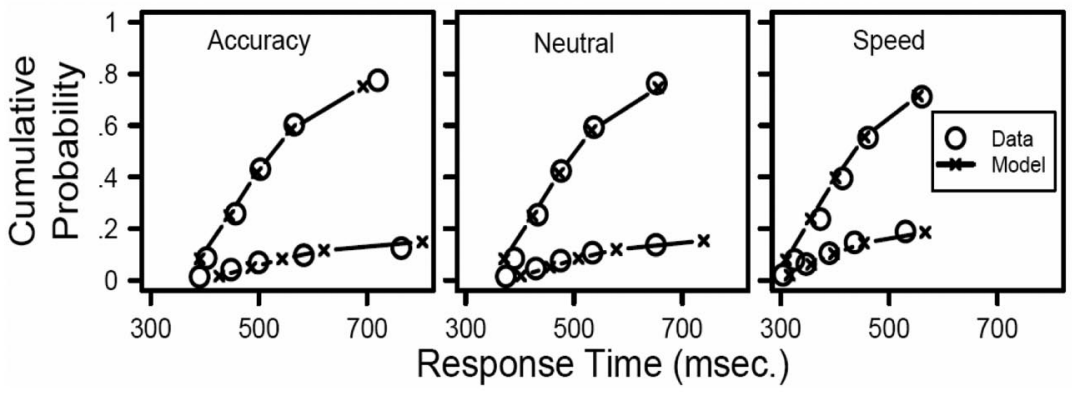

Evidence for

Left Response

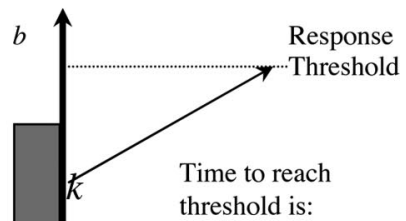

$(b-k) / d$

B

Young
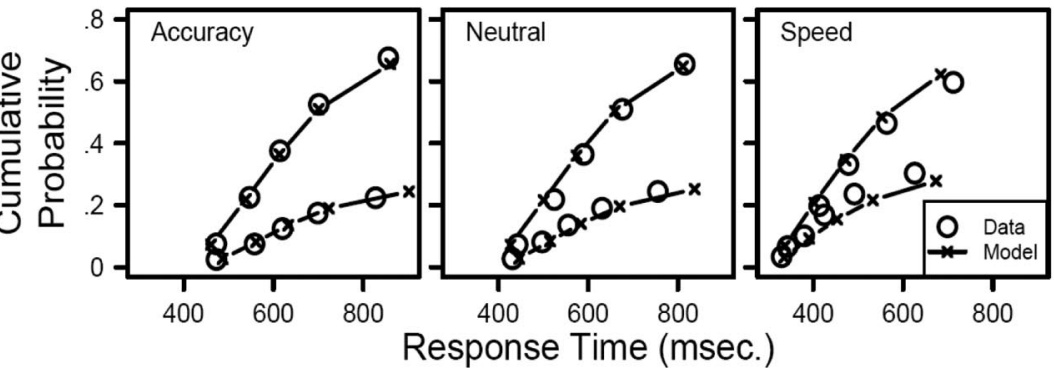

Figure 3. A, The LBA model. In the LBA model, the decision to response either "left" or "right" is modeled as a race between two accumulators. Activation in each accumulator begins at a random point between zero and $A$ and increases at a rate that depends on the match between the stimulus and response. A response is given by whichever accumulator first reaches the threshold $b$, and the predicted response times depend on the time taken to reach that threshold. $\boldsymbol{B}$, Model fits. Quantiles estimated from data (circles) and predicted by the LBA model (crosses with lines) separately for young and elderly participants. The three panels show data from three different response caution conditions. Within each panel, the upper lines and symbols show quantile estimates for correct responses, and the lower set for incorrect responses. The data and model predictions were averaged across participants and across left vs right stimuli.

rates), but are less proficient at controlling their bias or response caution settings (the older group had significantly larger start point variability, parameter $A: p=$ 0.0007).

In this work the relation between aging and the SAT is of primary interest. To examine this relation, we used the LBA parameter estimates to quantify the extent to which participants successfully implemented the instruction to set a fast-buterror-prone speed-accuracy tradeoff setting. For each participant, we calculated a measure of response caution by taking the estimated threshold parameter for each of the three emphasis conditions (i.e., $b$ from the speed-emphasis, neutral-emphasis and accuracy-emphasis conditions) and subtracted from those half of the estimated starting point variability estimate (i.e., $A$ ). This measure gives the average distance that

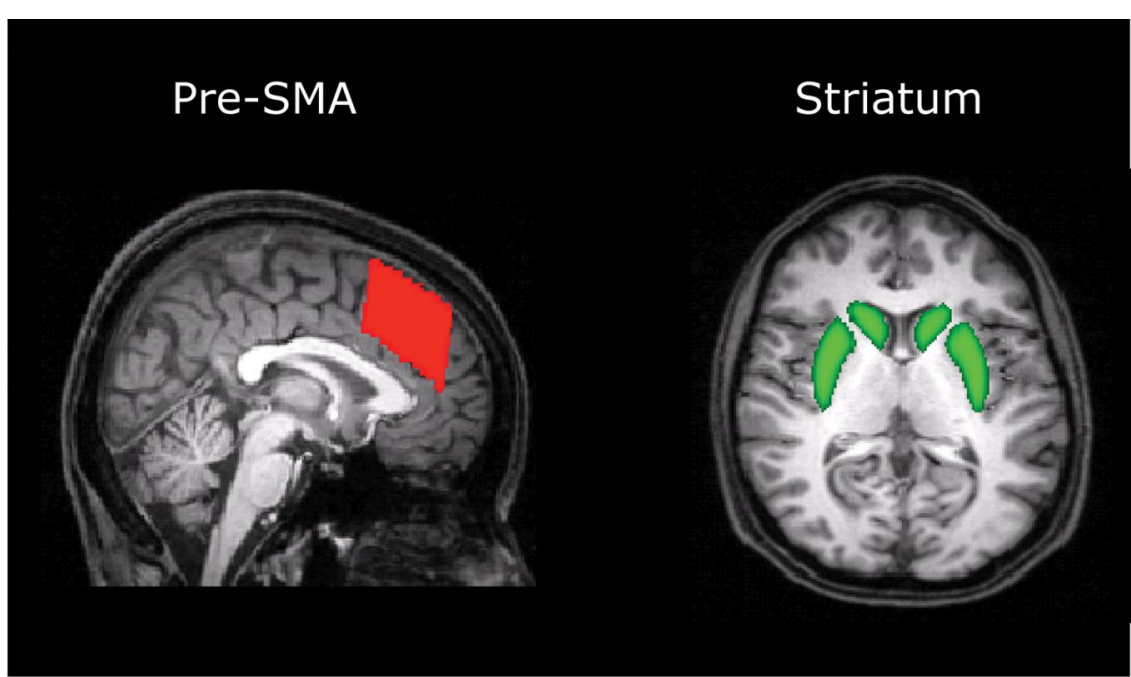

Figure 4. Target areas. Location of brain areas for which connectivity was quantified. 


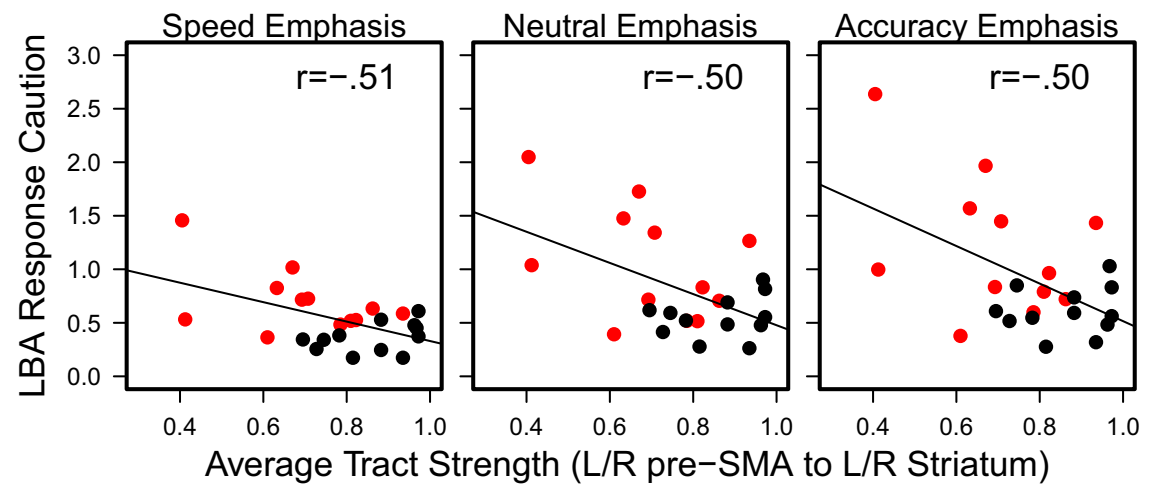

Figure 5. Tract strength vs response caution. Average tract strength vs response caution separately for three emphasis conditions (panels) for elderly (red) and young (black) participants.

an evidence accumulation process must cover to reach threshold. We note that similar results hold if response caution is measured in slightly different ways, such as using the ratio (rather than difference) of the two parameters, $b / A$, as used by Forstmann et al. (2008a). In the main analyses reported below, we summarize overall response caution for each participant using the average response caution measure across the three different emphasis conditions. We also repeated our analyses with the response caution measures calculated from each emphasis condition separately, and we note where those analyses differ from the main ones.

\section{Differences in tract strength}

To test the hypothesis of a main effect in tract strength between the pre-SMA and the striatum between elderly and young participants we computed four independent $t$ tests comparing young and elderly participants' tract strengths between the right preSMA connecting to the right striatum, the right pre-SMA to the left striatum, the left pre-SMA to the right striatum, and the left pre-SMA to the left striatum. These tests were motivated by previous studies that demonstrated the presence of both ipsilateral and contralateral connections between pre-SMA and striatum, in humans as well as monkeys (Inase et al., 1999; Johansen-Berg et al., 2004; Lehéricy et al., 2004).

Compared to the elderly, the young participants had stronger connections from right pre-SMA to the right striatum, $t_{(22)}=$ $2.84, p=0.01$, from right pre-SMA to left striatum, $t_{(22)}=3.38$, $p=0.003$, from left pre-SMA to right striatum, $t_{(22)}=3.02, p=$ 0.006 , and-albeit on the cusp of significance-from left preSMA to left striatum, $t_{(22)}=2.07, p=0.05$. Overall, these results are consistent with other work that has generally shown decreases in white matter integrity with age (Liston et al., 2006; Grieve et al., 2007; Bucur et al., 2008).

\section{Correlations between tract strength, age, and response caution}

The corticostriatal hypothesis makes specific and unidirectional predictions about correlations between tract strength, age, and response caution. Increased age should be associated with decreased tract strengths between the pre-SMA and striatum, and both quantities should be associated with increased response caution. We investigated these predictions using standard Pearson correlation coefficients, with one-tailed $t$ tests to assess statistical reliability. In all cases, we measured response caution as described above (using $b-A / 2$ ) and collapsed across emphasis conditions to have a single measure per subject. However, we repeated all analyses with different response caution measures (both $b$ alone and $b / A$ ) and also separately for each emphasis condition, and obtained similar results. We also ran the analyses twice: once with the four tract strength measurements from left/right pre-SMA to left/right striatum averaged into a single measurement, and once where they were kept separate. These results were mostly similar, so only the simpler averaged version is described below, except where differences were found.

\section{Age and response caution}

There was a reliable correlation between age and average response caution $(r=$ $0.57, p=0.002$ ), and similar results were obtained when response caution measures from the three emphasis conditions were considered separately (speed $r=0.63, p=0.001$; neutral $r=0.54, p=0.003$; accuracy $r=0.53, p=0.004)$. Very similar results were obtained when these analyses were restricted to just the younger participants (for the average correlation, $r=0.53, p=0.004$ ) but there was no reliable correlation within just the elderly group $(p>0.2)$.

\section{Age and tract strength}

There was a significant negative correlation between age and the average tract strength across the four tracts of interest $(r=-0.51$, $p=0.005$ ), and this was confirmed separately for each tract (correlations ranged from -0.39 to -0.57 , with all $p$ values smaller than .030). The observed ranges of age and tract strengths were too severely restricted to observe these correlations separately within the elderly and younger groups.

\section{Tract strength and response caution}

The simplest analysis is the one where response caution measures were averaged across the three emphasis conditions, and where the four tract strength measurements were averaged together. This analysis revealed a significant negative correlation $(r=$ $-0.51, p=0.01$ ), that was also marginally significant when just considering the elderly participants $(r=-0.44, p=0.07)$, but was not significant for the younger group on their own. When the four tract strength measurements were considered separately, very similar results were obtained. The strongest correlations for the single tract measurement were observed for tracts emanating from the right pre-SMA ( $r=-0.52$ to right striatum and $r=$ -0.51 to left striatum, both $p$ values $=0.005)$, but the correlations for the tracts emanating from the left pre-SMA were still reliable ( $r=-0.49$ to right striatum and $r=-0.46$ to left striatum, both $p$ values $<0.012$ ).

Returning to the averaged tract strength measure, but this time considering separately the response caution measures from the three emphasis conditions, we again found results consistent with the corticostriatal hypothesis: within each emphasis condition, results similar to the average were obtained. The correlations between response caution and the averaged tract strength measures were as follows: for the speed-emphasis condition, $r=$ $-0.51, p=0.006$; for neutral-emphasis $r=-0.50 p=0.006$; for the accuracy-emphasis condition $r=-0.50, p=0.006$. These three correlations are represented graphically in Figure 5, which plots average tract strength (on the $x$-axis) against response caution (on the $y$-axis), separately for the three emphasis conditions (panels). In each panel, the red and black symbols represent the elderly and younger participants, respectively, and the black line shows the predicted relationship from the correlation. 


\section{A control analysis}

Our data support the corticostriatal hypothesis that response caution is closely related to age and white matter connectivity in particular tracts. However, there is an alternative, and simpler, hypothesis that we would like to assess. It is possible instead that our measurements of tract strength simply provide anotherand possibly better-way of measuring age. That is, contrary to our hypothesis, the proposed corticostriatal network might have little to do with setting decision caution, but nevertheless measurements of connectivity in this network provide accurate measurements of general system-wide degeneration that occurs with age.

To assess this hypothesis, we calculated the strength of the tract connecting the left and right pre-SMA in each individual. According to our hypothesis, this connection should not play a role in setting decision caution, so it should not explain variance in LBA response caution above and beyond that captured by general aging processes. We used this tract strength measurement in place of the tracts connecting the pre-SMA and striatum in the analyses above, to assess whether the measurement of any plausible tract might give the same results. We observed differences in the results that were more consistent with the corticostriatal hypothesis than with some hypothesis of general system-wide degradation. For example, whereas the previous tract strength measurements all had significant correlations with average response caution, the tract connecting left with right pre-SMA did not $(r=-0.24, p=0.13)$. This result is unlikely to be due to the control tract simply being measured with greater noise, as this tract still showed a moderate negative correlation with age $(r=$ $-0.31, p=0.074)$. Nevertheless, it should be noted that the difference in correlations ( $r=-0.51$ for the average corticostriatal tracts versus $r=-0.24$ for the tract from right to left pre-SMA) was only marginally significant ( $p=0.09$, permutation test), so that the results are suggestive but not definitive.

\section{Discussion}

Mathematical modeling of the behavioral data confirmed that, when instructed to respond quickly, elderly participants do not lower their response thresholds as much as young participants (Starns and Ratcliff, 2010). The traditional explanation of this phenomenon ascribes the aversion of elderly participants to adopt fast speed-accuracy tradeoff settings to a strategic choice that is entirely under voluntary control. Here we proposed and explored a radically different account, one that ascribes the aversion for fast speed-accuracy tradeoff settings to structural limitations in brain connectivity.

The motivation for this alternative account came from the striatal hypothesis of the SAT (Forstmann et al., 2008; Bogacz et al., 2010). The striatal hypothesis holds that speed stress increases the input from cortex to striatum; activation of the striatum disinhibits the cortex and facilitates faster but possibly premature responses. Within this framework, the age-related slowing that is due to more conservative speed-accuracy tradeoff settings might not reflect a strategic choice but rather the degeneration of white matter connections between cortex and striatum.

Consistent with the striatal hypothesis of the SAT, our results showed that older adults have relatively weak white matter connections between pre-SMA and striatum (cf. Voineskos et al., 2010). According to the striatal hypothesis of the SAT, these two sets of seemingly unrelated results are in fact causally related, as the control over threshold settings is assumed to be instantiated via the corticostriatal network. We should acknowledge that the present findings are not uniquely consistent with the striatal hy- pothesis of SAT; that is, advancing age may cause both conservative speed-accuracy tradeoff settings and, through a different mechanism, degeneration of corticostriatal connections. In theory, this alternative explanation could be evaluated by means of a mediation analysis, in which one could test the hypothesis that weak corticostriatal connections result in conservative threshold settings, independent of age. Such an analysis requires a much larger sample size, and, importantly, a broader range of age. The ages of participants in our study were tightly controlled-by design - but they are thus too homogeneous and too small to draw any firm conclusions about mediation. Nevertheless, the striatal hypothesis is parsimonious and offers a novel perspective on why elderly participants prefer conservative thresholds; moreover, the main prediction from the striatal hypothesis was supported by the data. Nevertheless, the current data do not compel an explanation in terms of the striatal hypothesis.

The outcome of this study is in line with at least two slightly different explanations of why older adults are relatively reluctant to adjust their response thresholds. The first explanation holds that it is difficult for older adults to adopt risky response thresholds because the brain mechanism that allows such threshold adjustment (i.e., the increased activation along the corticostriatal pathway) has been compromised. According to this explanation, older adults are relatively cautious not because they deliberately choose to engage in further information accumulation, but because their brains lack an efficient mechanism to shortcut the process of information accumulation. The second explanation holds that older adults could indeed choose to adopt risky response thresholds, but that they avoid doing so because of the consequences: the use of risky response thresholds means that one is operating on a very sensitive part of the SAT curve, where a small decrease in RT can lead to a large drop in accuracy. It may be that the relatively weak corticostriatal connections in the elderly do not support the tight control over the SAT that is needed to effectively manage performance in the more risky SAT ranges, so that older adults are wise to avoid them (for behavioral support for this assertion see Smith and Brewer, 1985, 1995).

The control of SAT is an important determinant of age-related slowing, and our work constitutes a new direction toward a more detailed and neuroscientific understanding of the challenges that older adults face when they are put under time pressure. Specifically, our work corroborates and generalizes the hypothesis that the pre-SMA in concert with the striatum supports the flexible adjustment of response caution (Forstmann et al., 2008, 2010a; Bogacz et al., 2010). Furthermore, our work suggests the possibility that older adults are inflexible and cautious not just by voluntary choice, but at least partially by the structural limitations of corticostriatal white matter connections.

\section{References}

Aron AR, Behrens TE, Smith S, Frank MJ, Poldrack RA (2007) Triangulating a cognitive control network using diffusion-weighted magnetic resonance imaging (MRI) and functional MRI. J Neurosci 27:3743-3752.

Beaulieu C (2009) The biological basis of diffusion anisotropy. In: Diffusion MRI: from quantitative measurement to in vivo neuroanatomy (Johansen-Berg H, Behrens TEJ, eds), pp 105-126. London: Elsevier.

Behrens TE, Johansen-Berg H, Woolrich MW, Smith SM, Wheeler-Kingshott CA, Boulby PA, Barker GJ, Sillery EL, Sheehan K, Ciccarelli O, Thompson AJ, Brady JM, Matthews PM (2003) Non-invasive mapping of connections between human thalamus and cortex using diffusion imaging. Nat Neurosci 6:750-757.

Behrens TE, Johansen-Berg H, Jbabdi S, Rushworth MF, Woolrich MW (2007) Probabilistic diffusion tractography with multiple fibre orientations: what can we gain? Neuroimage 34:144-155. 
Bogacz R, Wagenmakers EJ, Forstmann BU, Nieuwenhuis S (2010) The neural basis of the speed-accuracy tradeoff. Trends Neurosci 33:10-16.

Brinley JF (1965) Cognitive sets, speed and accuracy of performance in the elderly. In: Behavior, aging and the nervous system (Welford AT and Birren JE, eds), pp. 114-149. Springfield, IL: Thomas.

Britten KH, Shadlen MN, Newsome WT, Movshon JA (1992) The analysis of visual motion: a comparison of neuronal and psychophysical performance. J Neurosci 12:4745-4765.

Brown SD, Heathcote A (2008) The simplest complete model of choice response time: linear ballistic accumulation. Cogn Psychol 57:153-178.

Bucur B, Madden DJ, Spaniol J, Provenzale JM, Cabeza R, White LE, Huettel SA (2008) Age-related slowing of memory retrieval: contributions of perceptual speed and cerebral white matter integrity. Neurobiol Aging 29:1070-1079.

Burnham KP, Anderson DR (2002) Model selection and multimodel inference: a practical information theoretic approach, Ed 2. Springer: New York.

Cerella J (1985) Information processing rates in the elderly. Psychol Bull 98:67-83.

Cohen MX, Schoene-Bake JC, Elger CE, Weber B (2009) Connectivitybased segregation of the human striatum predicts personality characteristics. Nat Neurosci 12:32-34.

Donkin C, Brown SD, Heathcote A (2011) Drawing conclusions from choice response time models: a tutorial using the Linear Ballistic Accumulator. J Math Psychol 55:140-151.

Fields RD (2008) White matter in learning, cognition and psychiatric disorders. Trends Neurosci 31:361-370.

Forstmann BU, Dutilh G, Brown S, Neumann J, von Cramon DY, Ridderinkhof KR, Wagenmakers EJ (2008) Striatum and pre-SMA facilitate decision-making under time pressure. Proc Natl Acad Sci USA 105:17538-17542.

Forstmann BU, Brown S, Dutilh G, Neumann J, Wagenmakers EJ (2010a) The neural substrate of prior information in perceptual decision making: a model-based analysis. Front Hum Neurosci 21:1-12.

Forstmann BU, Anwander A, Schäfer A, Neumann J, Brown S, Wagenmakers EJ, Bogacz R, Turner R (2010b) Cortico-striatal connections predict control over speed and accuracy in perceptual decision making. Proc Natl Acad Sci U S A 107:15916-15920.

Gold JI, Shadlen MN (2007) The neural basis of decision making. Annu Rev Neurosci 30:535-574.

Grieve SM, Williams LM, Paul RH, Clark CR, Gordon E (2007) Cognitive aging, executive function, and fractional anisotropy: a diffusion tensor MR imaging study. AJNR Am J Neuroradiol 28:226-235.

Inase M, Tokuno H, Nambu A, Akazawa T, Takada M (1999) Corticostriatal and corticosubthalamic input zones from the presupplementary motor area in the macaque monkey: comparison with the input zones from the supplementary motor area. Brain Res 833:191-201.

Johansen-Berg H, Behrens TE, Robson MD, Drobnjak I, Rushworth MF, Brady JM, Smith SM, Higham DJ, Matthews PM (2004) Changes in connectivity profiles define functionally distinct regions in human medial frontal cortex. Proc Natl Acad Sci U S A 101:13335-13340.

Lehéricy S, Ducros M, Krainik A, Francois C, Van de Moortele PF, Ugurbil K, Kim DS (2004) 3-D diffusion tensor axonal tracking shows distinct SMA and pre-SMA projections to the human striatum. Cereb Cortex 14:1302-1309.

Liston C, Watts R, Tottenham N, Davidson MC, Niogi S, Ulug AM, Casey B] (2006) Frontostriatal microstructure modulates efficient recruitment of cognitive control. Cereb Cortex 16:553-560.

Mink JW (1996) The basal ganglia: focused selection and inhibition of competing motor programs. Prog Neurobiol 50:381-425.

Nelder JA, Mead R (1965) A simplex algorithm for function minimization. Comput J 7:308-313.

Oldfield RC (1971) The assessment and analysis of handedness: The Edinburgh Inventory. Neuropsychologia 9:97-113.

Pew RW (1969) The speed-accuracy operating characteristic. Acta Psychol 30:16-26.

Rabbitt P (1979) How old and young subjects monitor and control responses for accuracy and speed. Br J Psychol 70:305-311.

Ratcliff R, Rouder JN (1998) Modeling response times for two-choice decisions. Psychol Sci 9:347-356.

Ratcliff R, Thapar A, McKoon G (2007) Application of the diffusion model to two-choice tasks for adults 75-90 years old. Psychol Aging 22:56-66.

Salthouse TA (1979) Adult age and the speed-accuracy trade-off. Ergonomics 22:811-821.

Salthouse TA (1996) The processing-speed theory of adult age differences in cognition. Psychol Rev 103:403-428.

Scholz J, Klein MC, Behrens TE, Johansen-Berg H (2009) Training induces changes in white-matter architecture. Nat Neurosci 12:1370-1371.

Schwarz G (1978) Estimating the dimension of a model. Ann Stat 6:461-464.

Smith GA, Brewer N (1985) Age and individual differences in correct and error reaction times. Br J Psychol 76:199-203.

Smith GA, Brewer N (1995) Slowness and age: speed-accuracy mechanisms. Psychol Aging 10:238-247.

Smith Y, Bevan MD, Shink E, Bolam JP (1998) Microcircuitry of the direct and indirect pathways of the basal ganglia. Neuroscience 86:353-387.

Starns JJ, Ratcliff R (2010) The effects of aging on the speed-accuracy compromise: boundary optimality in the diffusion model. Psychol Aging 25:377-390

Tuch DS, Salat DH, Wisco JJ, Zaleta AK, Hevelone ND, Rosas HD (2005) Choice reaction time performance correlates with diffusion anisotropy in white matter pathways supporting visuospatial attention. Proc Natl Acad Sci U S A 102:12212-12217.

Voineskos AN, Rajji TK, Lobaugh NJ, Miranda D, Shenton ME, Kennedy JL, Pollock BG, Mulsant BH (2010) Age-related decline in white matter tract integrity and cognitive performance: a DTI tractography and structural equation modeling study. Neurobiol Aging. Advance online publication. Retrieved May 3, 2011. doi:10.1016/j.neurobiolaging.2010.02.009. 